\title{
DETERMINATION OF COMPLEX DIELECTRIC PERMITTIVITY OF LOSS MATERIALS AT MICROWAVE FREQUENCIES
}

\author{
U.C. Hasar, M.F. Akay and S.N. Kharkovsky \\ Department of Electrical and Electronics Engineering, \\ Cukurova University, Adana, 01330, Turkey \\ ugurcem@eemb.cu.edu.tr
}

\begin{abstract}
The method of determination of complex dielectric permittivity of loss materials at microwave frequencies (X-band) using measured amplitudes of reflection and transmission coefficients and numerical calculations is developed. Different numerical methods namely graphical, bisection (halving), newton, and secant, are applied in order to determine the permittivity of cement-based materials. Simulation time and errors of these methods are compared. It is shown that the fastest and most accurate method is the bisection (halving) method because it is a global method.
\end{abstract}

Keywords- Complex dielectric permittivity of loss materials, root-finding and graphical methods, cement-based materials, microwave measurement.

\section{INTRODUCTION}

Dielectric properties, usually referred to permittivity, are intrinsic properties that describe wave-matter interaction and, therefore, are dependent on contents of the material, for example: moisture content, density and temperature. Cement-based materials (cement paste, mortar, concrete etc.) are widely used in many structures of the construction industry. For that reason, the knowledge of dielectric properties of such materials is very important for both propagating related research, for example: microwave propagation modelling to develop indoor wireless communication systems [4,7], and quality and content of these materials against time.

Free-space microwave techniques have been widely used for dielectric property measurements particularly since recent advances in microwave components and instrumentation made them more convenient. Complex permittivity of samples can be determined from measured reflection and/or transmission coefficients using microwave techniques [2,4,5,8,9] and suitable numerical methods [3]. The graphical method, the first applied method, can be applied for the problems with more than one independent variable [2,5]. However, its simulation time is high and its precision is low. To reduce the simulation time and to increase the precision, bisection, newton, and secant methods [3] are tested.

In this paper, the complex permittivity of loss specimens by using only the amplitudes of reflection and transmission coefficients at microwave frequencies (Xband) is determined. Then, the numerical methods used for the determination of the complex permittivities of loss samples are compared. Finally, an error analysis for both measurement system and numerical methods are presented.

\section{THEORY}

A typical situation in the measurement of the permittivity of a sample using the free-space technique is shown in Figure1. 


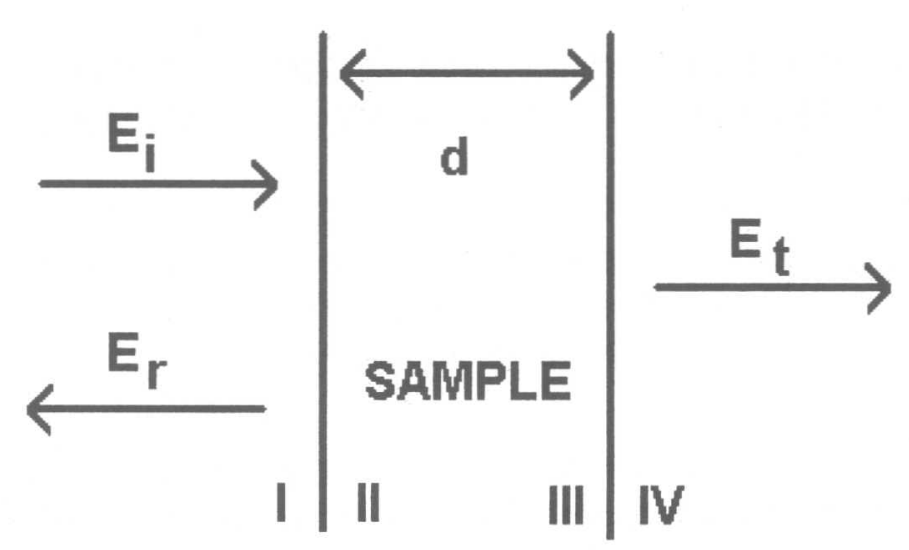

Figure 1. Typical situation in the measurement of permittivity of a sample.

The wave travels from the radiating antenna to the receiving antenna through the two different media, air and sample. Reflection occurs at the interface of the air-sample I, and multiple reflections occur between each sides of the sample.

For loss materials, the expressions for reflection coefficient, $r$, and transmission coefficient, $t$, can be expressed as [1]

$$
\begin{aligned}
& r=r_{12} \\
& t=\left(1-r_{12}^{2}\right) e^{-j \Theta}
\end{aligned}
$$

where

$$
k_{o}=\frac{2 \pi}{\lambda_{o}}, \quad \Theta=k_{s} d, \quad k_{s}=k_{o} \sqrt{\varepsilon}, \quad \varepsilon=\varepsilon^{\prime}-j \varepsilon " .
$$

In the foregoing equations in (1) and (2), $\lambda_{o}, d$, and $\varepsilon$ are the wavelength in free-space, thickness and dielectric permittivity of the material, respectively.

In experimental techniques, the amplitudes of reflection and transmission coefficients $|r|$ and $|t|$ are measured in decibels, defined as

$$
T=-20 \log |t|, \quad R=-20 \log |r| \text {. }
$$

In order to find the permittivity, equations in (1) should be solved together. To simplify the solution of the equations, let $\sqrt{\varepsilon}=a-j b$. After this manipulation, a nonlinear equation that has a dependence only on $a$ can be found as

$$
f(a)=\sqrt{2 a\left(1-|r|^{4}\right)-\left(1-|r|^{2}\right)^{2}} \exp \left\{-\Phi \sqrt{\frac{2 a\left(1+|r|^{2}\right)}{\left(1-|r|^{2}\right)}-\left(1+a^{2}\right)}\right\}-|t| a=0
$$

where

$$
\Phi=k_{o} d \text {. }
$$

The root of the function, $a$, can be computed using appropriate numerical methods. After finding the value of $a$, the value of $b$ can be calculated by $b=\sqrt{\frac{(1-a)^{2}-(1+a)^{2}|r|^{2}}{|r|^{2}-1}}$. 
After all, the real and imaginary parts of the permittivity can then be found $\mathrm{b}$ using the following simple formulas

$$
\varepsilon^{\prime}=a^{2}-b^{2} \quad \text { and } \quad \varepsilon^{\prime \prime}=2 a b .
$$

\section{MEASUREMENT SYSTEM}

Typical loss cement-based structures with different contents, and dimensions were prepared and used for investigation of permittivity values at X-band $(8-12 \mathrm{GHz})$ by using free space method. There are two main parts of the proposed microwave measurement system: microwave and control parts, respectively. The microwave part of the measurement system is used to determine the amplitudes of incident, reflected and transmitted waves as three separate parameters, and the main purpose of the control circuit is to provide communication between the microwave experimental set-up and the computer. The detailed information about microwave measurement set-up and control circuit set-up can be found in [5] and [6] respectively.

\section{NUMERICAL CALCULATIONS WITH DIFFERENT METHODS}

\subsection{Determination of Permittivity}

Appropriate numerical methods are developed to determine the dielectric permittivities of loss specimens by using only the amplitudes of reflection and transmission coefficients, $|r|$ and $|t|$, respectively. These are graphical method and rootfinding methods namely, bisection method, newton method and secant method.

We firstly applied graphical method to determine the complex permittivity of samples. The chart for this method is shown in Figure 2.

In this method, each prospective value of real and imaginary parts of the permittivity is put into equations in (1), then the results are matched with the input parameters. After that, constant value lines of reflection and transmission coefficients (CR and CT) for the values of the amplitudes of the reflection and transmission coefficients (Rmea and Tmea) are obtained. In Figures (4) and (5) are the constant value lines for Rmea and Tmea shown, respectively. In the calculation, the frequency $f=10.38 \mathrm{GHz}$ and the sample thickness $d=150 \mathrm{~mm}$.

For loss media case, there is just one cross point between the lines CR and CT [2] esulting in the complex permittivity of loss sample. The cross point can found by utting the CR onto CT. The result is shown in Figure 6. To find the complex rmittivity, a permittivity finding algorithm is employed. The results of this algorithm z given in Table 1 .

Secondly, bisection, newton, and secant methods are employed. The general chart each of these methods is shown in Figure 3. The purpose of all of these root-finding hods is to find a domain. Not only should the domain support a suitable region, but it should reduce the time for determining the complex permittivity. For that reason, ipplied an algorithm for finding the best region that complies with the foregoing rements. The results of the determined complex permittivity values are larised in Table 1.

As is clearly seen from Table 1, the results obtained from the applications of each 'nt algorithm are in good agreement except that the graphical method has the tivity values slightly different from the other values. 


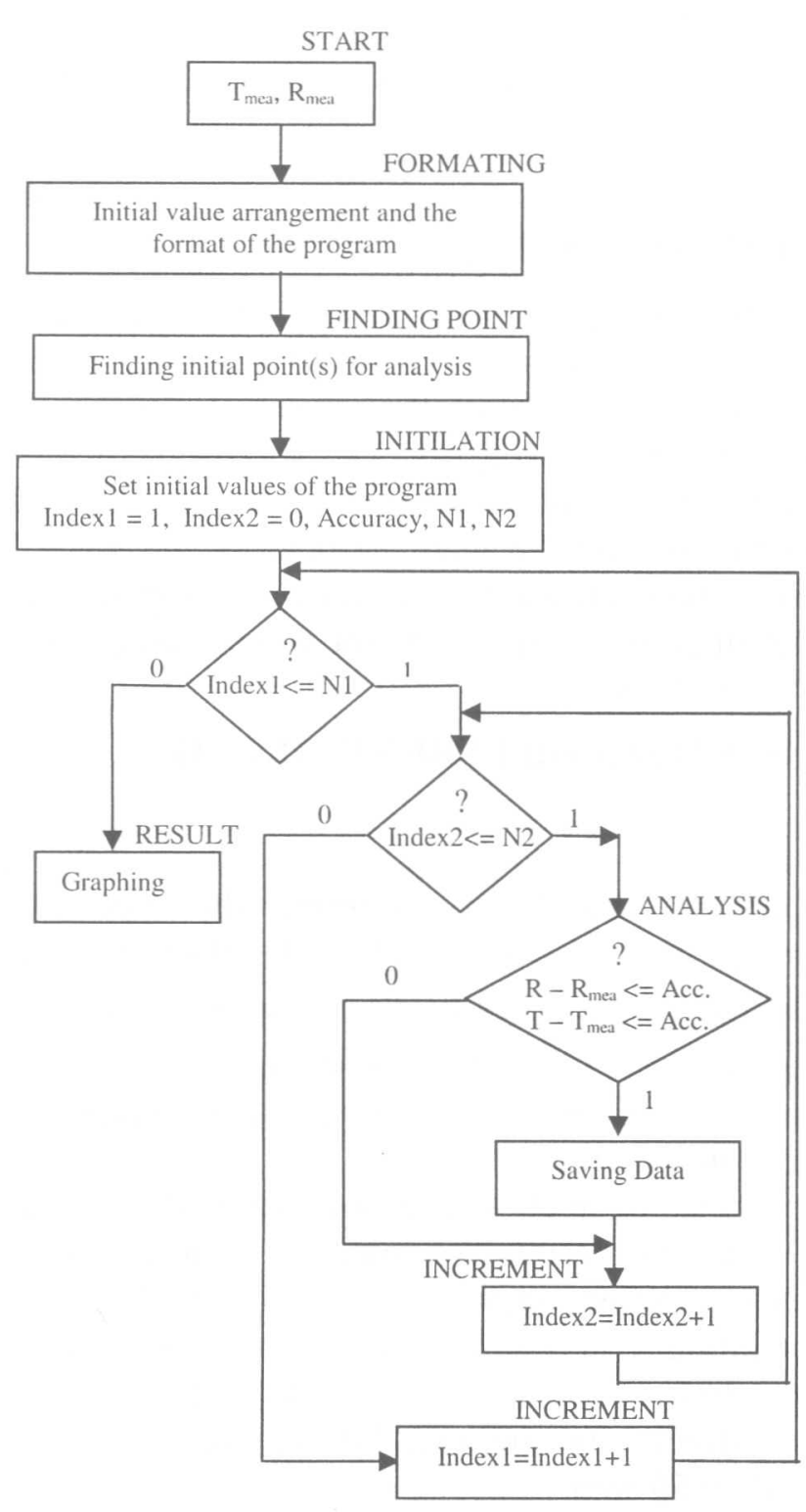

Figure 2. The ASM chart for graphical method.

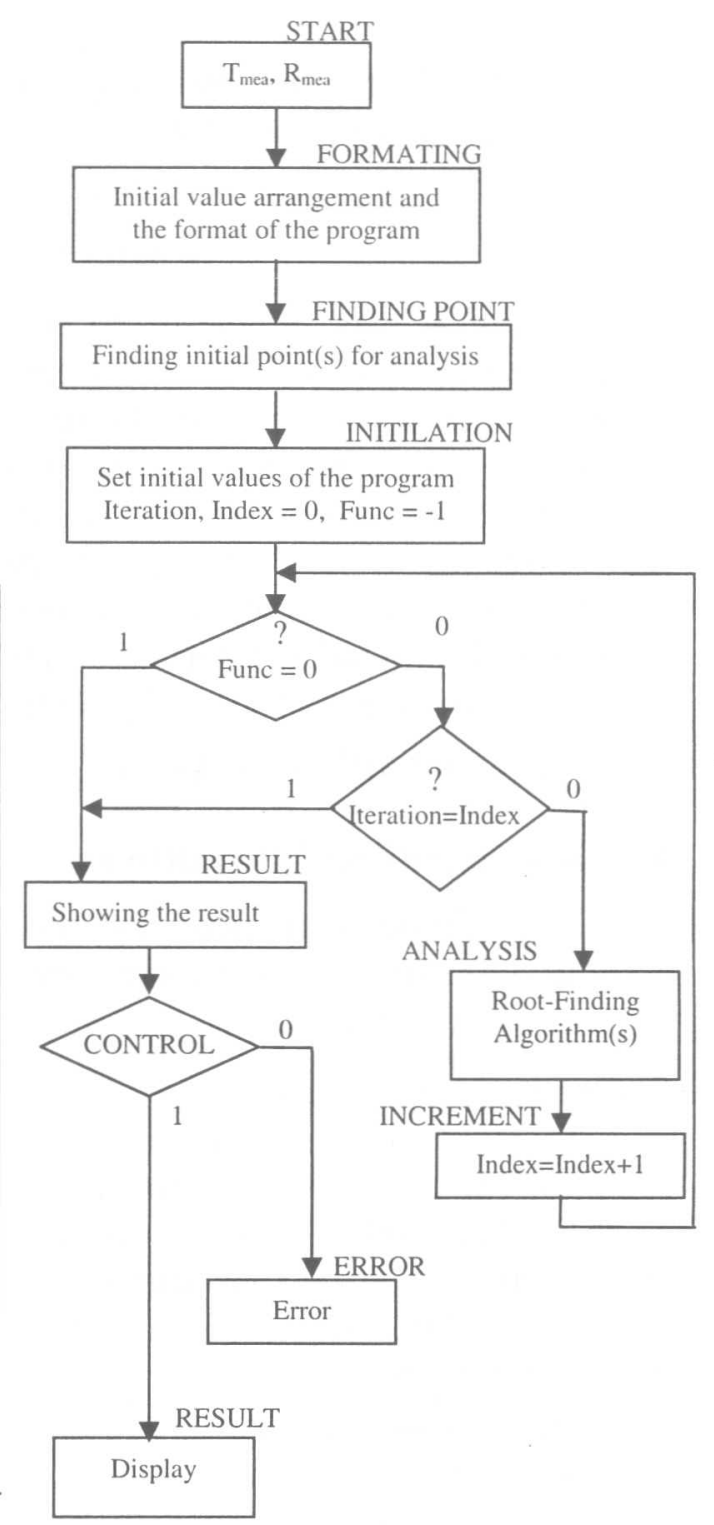

Figure 3. The general ASM chart for root-finding methods

For all methods, the error degree value is put the same. It is observed that the bisection method produces results faster than the others. The bisection method is called global method because the starting point information need not be accurate at all. Besides, the newton and regula falsi methods are called local methods because their models and assumptions apply only near the solutions. Therefore, the simulation time for newton and secant methods is slightly greater than that of bisection method although they are faster than bisection method. As is seen from the Table 1 that the simulation time for graphical method is much longer than the simulation times of other methods. The reason is that the domain of graphical method is much broader than the domains of others. 


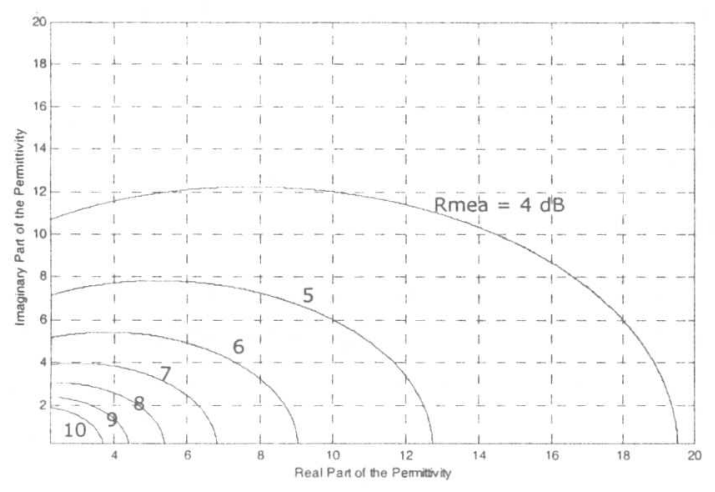

Figure 4. The constant value lines for different values of Rmea (CR).

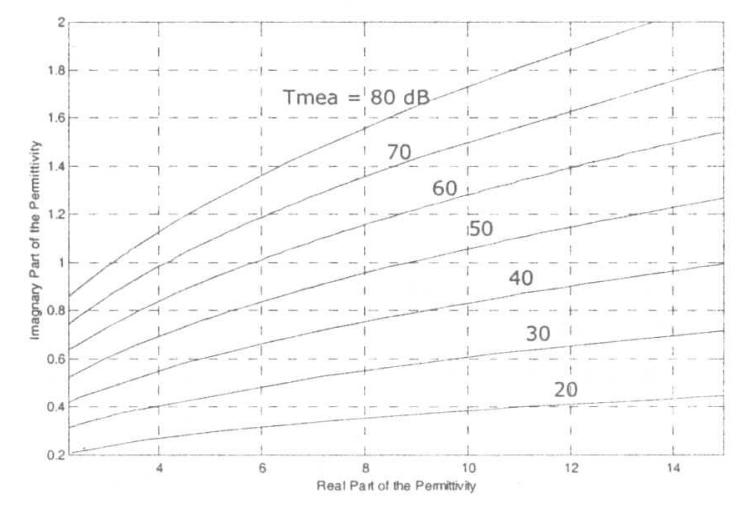

Figure 5. The constant value lines for different values of Tmea (CT).

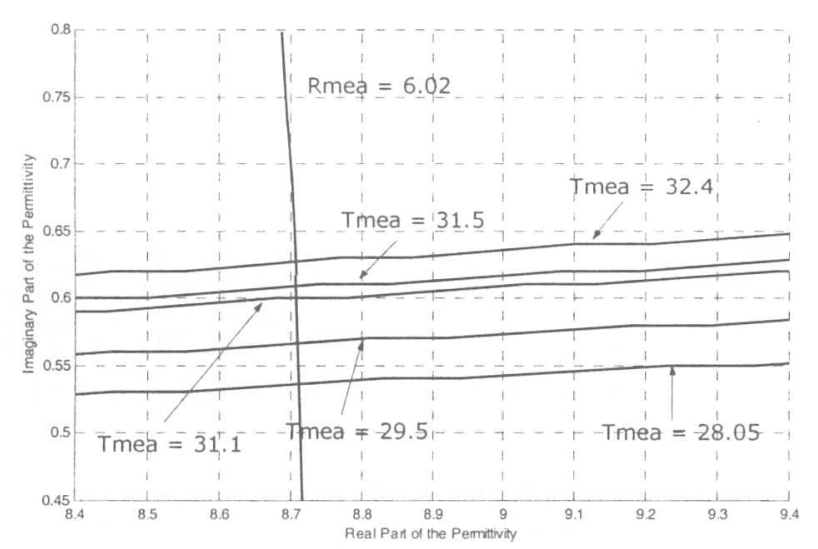

Figure 6. The result of the combination of constant value lines for Rmea (CR) and Tmea (CT).

Table 1. Estimated permittivity values for four different methods with input parameters, frequency $f=10.38 \mathrm{GHz}$ and the sample thickness $d=150 \mathrm{~mm}$.

\begin{tabular}{|c|c|c|c|c|c|}
\hline \multicolumn{2}{|c|}{ Input values $(\mathrm{dB})$} & \multicolumn{4}{|c|}{ Complex permittivity values } \\
\hline $\mathrm{T}$ & $\mathrm{R}$ & Bisection & Newton & Secant & Graphical \\
\hline 32.4 & \multirow{5}{*}{$\frac{n}{ \pm}$} & $7.272-0.578 \mathrm{i}$ & $7.272-0.577 i$ & $7.272-0.575 i$ & $7.142-0.562 \mathrm{i}$ \\
\hline 31.5 & & $7.274-0.561 \mathrm{i}$ & $7.274-0.560 \mathrm{i}$ & $7.274-0.563 \mathrm{i}$ & $7.145-0.544 i$ \\
\hline 31.1 & & $7.275-0.554 i$ & $7.275-0.555 i$ & $7.275-0.555 i$ & $7.147-0.533 i$ \\
\hline 29.5 & & $7.279-0.523 \mathrm{i}$ & $7.279-0.522 i$ & $7.279-0.523 i$ & $7.150-0.506 i$ \\
\hline 28.05 & & $7.282-0.496 i$ & $7.282-0.498 i$ & $7.281-0.498 \mathrm{i}$ & $7.151-0.482 i$ \\
\hline 32.4 & \multirow{5}{*}{ ठ઼ } & $8.964-0.633 i$ & $8.964-0.631 \mathrm{i}$ & $8.964-0.633 i$ & $8.870-0.615 i$ \\
\hline 31.5 & & $8.966-0.614 i$ & $8.966-0.612 \mathrm{i}$ & $8.966-0.614 i$ & $8.878-0.603 \mathrm{i}$ \\
\hline 31.1 & & $8.967-0.605 i$ & $8.967-0.606 i$ & $8.967-0.606 i$ & $8.887-0.587 \mathrm{i}$ \\
\hline 29.5 & & $8.970-0.571 \mathrm{i}$ & $8.970-0.574 i$ & $8.971-0.568 \mathrm{i}$ & $8.890-0.550 \mathrm{i}$ \\
\hline 28.05 & & $8.974-0.541 \mathrm{i}$ & $8.974-0.539 i$ & $8.973-0.542 i$ & $8.895-0.516 \mathrm{i}$ \\
\hline \multicolumn{2}{|c|}{$\begin{array}{c}\text { Average } \\
\text { simulation time } \\
\text { (seconds) }\end{array}$} & 0.032 & 0.951 & 0.301 & 50.3 \\
\hline
\end{tabular}




\subsection{Comparison of the Results}

To investigate the suitability of the developed chart and the applied methods, w used measured values of $|r|$ and $|t|$, given for an operating frequency of $57.5 \mathrm{GHz}$ anc for a mortar sample having a thickness of $30 \mathrm{~mm}$ in [4], as input values to the programs and estimated the permittivity. The permittivity values in [4] corresponding to the measured values of $|r|$ and $|t|$ were estimated by using the non-linear least squares method. The comparison of the results and the percentage differences between them are shown in Tables 2 and 3, respectively. The percentage difference between real parts ranges from 0.001 to 0.438 and between imaginary parts ranges from 0.212 to 1.901 for root-finding methods, whereas the percentage difference between real parts ranges from 1.302 to 2.212 and between imaginary parts ranges from 1.613 to 2.192 for graphical method.

Table 2. Permittivity results for five different numerical methods, (*) stands for the permittivity values in [4].

\begin{tabular}{|c|c|c|c|c|}
\hline $\begin{array}{c}\text { Input Values } \\
(\mathrm{dB})\end{array}$ & $\begin{array}{c}\mathrm{T}=73.6, \\
\mathrm{R}=6.448\end{array}$ & $\begin{array}{c}\mathrm{T}=68.4, \\
\mathrm{R}=6.840\end{array}$ & $\begin{array}{c}\mathrm{T}=46.5, \\
\mathrm{R}=7.151\end{array}$ & $\begin{array}{c}\mathrm{T}=28.1, \\
\mathrm{R}=7.191\end{array}$ \\
\hline Bisection & $7.764-1.272 \mathrm{i}$ & $6.978-1.122 \mathrm{i}$ & $6.511-0.720 \mathrm{i}$ & $6.491-0.427 \mathrm{i}$ \\
\hline Newton & $7.764-1.272 \mathrm{i}$ & $6.978-1.122 \mathrm{i}$ & $6.511-0.728 \mathrm{i}$ & $6.494-0.420 \mathrm{i}$ \\
\hline Secant & $7.764-1.272 \mathrm{i}$ & $6.978-1.122 \mathrm{i}$ & $6.511-0.727 \mathrm{i}$ & $6.491-0.424 \mathrm{i}$ \\
\hline Graphical & $7.595-1.262 \mathrm{i}$ & $6.823-1.095 \mathrm{i}$ & $6.397-0.730 \mathrm{i}$ & $6.372-0.421 \mathrm{i}$ \\
\hline$\left(^{*}\right)$ & $7.731-1.283 \mathrm{i}$ & $6.978-1.113 \mathrm{i}$ & $6.483-0.714 \mathrm{i}$ & $6.495-0.428 \mathrm{i}$ \\
\hline
\end{tabular}

Table 3. Percentage differences for $\varepsilon^{\prime}$ and $\varepsilon^{\prime \prime}$ due to the results in [4].

\begin{tabular}{|c|c|c|c|c|c|c|c|c|c|}
\hline \multicolumn{2}{|c|}{ Input Values(dB) } & \multicolumn{2}{c|}{ Bisection } & \multicolumn{2}{c|}{ Newton } & \multicolumn{2}{c|}{ Secant } & \multicolumn{2}{c|}{ Graphical } \\
\hline $\mathrm{T}$ & $\mathrm{R}$ & $\Delta \varepsilon^{\prime}$ & $\Delta \varepsilon^{\prime \prime}$ & $\Delta \varepsilon^{\prime}$ & $\Delta \varepsilon^{\prime \prime}$ & $\Delta \varepsilon^{\prime}$ & $\Delta \varepsilon^{\prime \prime}$ & $\Delta \varepsilon^{\prime}$ & $\Delta \varepsilon^{\prime \prime}$ \\
\hline 73.6 & 6.45 & 0.419 & 0.857 & 0.420 & 0.881 & 0.420 & 0.878 & 1.741 & 1.613 \\
\hline 68.4 & 6.84 & 0.001 & 0.775 & 0.001 & 0.765 & 0.002 & 0.793 & 2.212 & 1.642 \\
\hline 46.5 & 7.15 & 0.437 & 0.831 & 0.435 & 1.892 & 0.438 & 1.755 & 1.302 & 2.192 \\
\hline 28.1 & 7.19 & 0.059 & 0.230 & 0.018 & 1.901 & 0.059 & 0.943 & 1.934 & 1.654 \\
\hline
\end{tabular}

\section{ERROR ANALYSIS}

Accuracy of determined complex permittivity values is critically based upon the :curacy of the applied methods and the accuracy of measured amplitudes of reflection $\mathrm{d}$ transmission coefficients.

Errors in microwave measurements have various natures and sources. The first in source of error in free-space measurement techniques is diffraction effects at the es of the sample. Creating the sample large enough can minimise diffraction at the es that it appears laterally infinite to the incident wave. The other main source of $\because$ is multiple reflections occurring inside the sample, between the sample interfaces the antennas and between the antennas through the sample. Those taking place 2 the sample can be avoided by selecting a thickness that ensures at least $10 \mathrm{~dB}$, ray attenuation. Figure 7 shows a simulation of the variation of attenuation, A with 
$\mathrm{d}$ at $10.38 \mathrm{GHz}$. The oscillations in amplitude, due to multiple reflections, are drastically reduced after the thickness of the sample is $8 \mathrm{~cm}$.

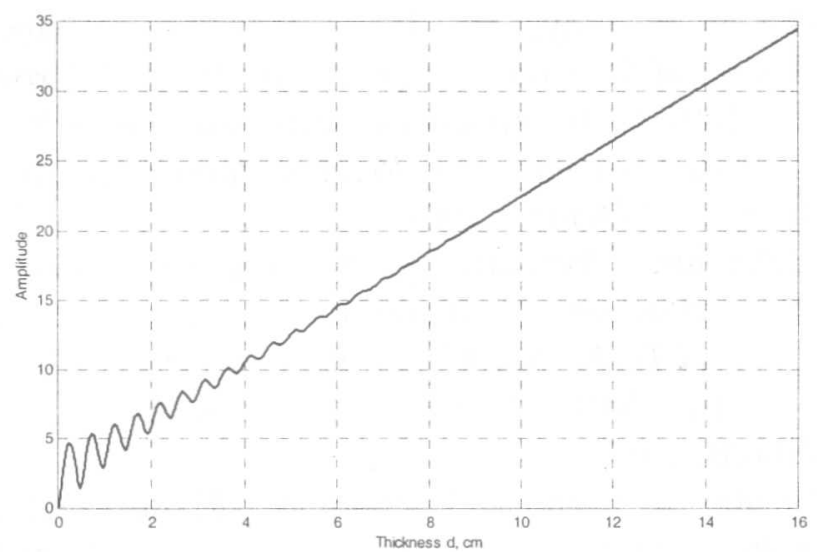

Figure 7. Simulation of the attenuation with different thicknesses. In the calculation,

$$
\varepsilon=8.96-0.633 \mathrm{i} \text { and } f=10.38 \mathrm{GHz} \text {. }
$$

The accuracy of applied methods highly depends on the accuracy of finding suitable domain and the root of (4) for root finding methods and the susceptibility of the program. The precision of the applied methods is calculated by inserting the found roots to the equation in (4), and then calculating the deviation of the results from zero. The calculated precision values are given in Table 4.

Table 4. Precisions of the applied methods with input parameters frequency $\mathrm{f}=10.38 \mathrm{GHz}$ and the sample thickness $\mathrm{d}=150 \mathrm{~mm}$.

\begin{tabular}{|c|c|c|c|c|}
\hline \multicolumn{2}{|c|}{ Input values } & \multicolumn{3}{|c|}{ Deviation from zero } \\
\hline $\mathrm{T}(\mathrm{dB})$ & $\mathrm{R}(\mathrm{dB})$ & Bisection & Newton & Secant \\
\hline 32.4 & \multirow{4}{*}{$\begin{array}{c}\text { n } \\
\text { İ } \\
\text { II } \\
\sim\end{array}$} & 0.000082 & 0.000808 & 0.000082 \\
\hline 31.5 & & 0.000049 & 0.000629 & 0.000049 \\
\hline 31.1 & & 0.000017 & 0.000341 & 0.000341 \\
\hline 29.5 & & $\cdot 0.000150$ & 0.001210 & 0.002002 \\
\hline
\end{tabular}

\section{CONCLUSIONS}

In this paper, numerical methods for the determination of complex permittivities of loss materials at microwave frequencies are applied, and their results are presented. It is shown that the results of applied numerical methods are in good agreement with the results of [4]. Although the graphical method is slower and has less accuracy, it can be used for giving the general view of both CT and CR and the intersection of them as well, and can be used if more than one independent variable in the formula exit. On the other hand, total independent variables must be reduced to one independent variable for root finding methods. If done, not only is the precision of these methods good, but also the simulation time of these methods is much less than that of the graphical method. In industrial based applications, precision and simulation time are the key features for microwave systems, and the simulation results show that bisection (halving) method is more suitable in these applications than other methods because bisection is a global method and does not require a special region. 
Acknowledgement- The authors would like to thank to the TUBITAK (The Scientific and Technical Research Council of Turkey) for supporting this work.

\section{REFERENCES}

[1] S. N. Kharkovsky, M. F. Akay, U. C. Hasar, C.D. Atiş, A Measurement System for the Determination of the Concrete Quality by Using Microwave Techniques, NIMIA-SC2001 - 2001 NATO Advanced Study Institute on Neural Networks for Instrumentation, Measurement, and Related Industrial Applications: Study Cases, Crema, Italy, 9-20 October 2001.

[2] Z.Ma, and S.Okamura, Permittivity determination using amplitudes of transmission and reflection coefficients at microwave frequency, IEEE Microwave Theory and Tech., vol. 47, no. 5, pp. 546-550, 1999.

[3] John R. Rice, Numerical Methods, Software, and Analysis, $3^{\text {rd }}$ ed. McGraw-Hill International Editions, 1987.

[4] K. Sato, T. Manabe, J. Polivka, T. Ihara V., Kasashima, and K. Yamaki, Measurement of the complex refractive index of concrete at $57.5 \mathrm{GHz}$, IEEE Trans. on the Antennas and Propagation, vol. 44, no.1, pp. 35-40, January 1996.

[5] S. N. Kharkovsky, M. F. Akay, U. C. Hasar, and C. D. Atis, Measurement and monitoring of microwave reflection and transmission properties of cement based specimens, Proc. Of $18^{\text {th }}$ IEEE.Instrum. Meas. Tech. Conf., vol. 1, pp. 513 518, May 2001.

[6] M. F. Akay, S. N. Kharkovsky, and U. C. Hasar, An automated amplitudes-only measurement system for permittivity determination using free-space method, Proc. of $18^{\text {th }}$ IEEE Instrum. Meas. Tech. Conf., vol. 1, pp. 503 - 506, May 2001.

[7] O.Landron, M.J.Feuerstein, T.S.Rappaport, A comparison of theoretical and empirical reflection coefficients for typical exterior wall surfaces in a mobile radio environment, IEEE Trans. Antennas Propagat., vol. 44, no. 3, pp. 341351, March 1996.

[8] Y. Huang and M. Nakhkash, Characterization of layered dielectric medium using reflection coefficient, Electronics Letters, vol. 34, no. 12, pp. 1207 1208, June 1998.

[9] S. Trabelsi, A. W. Kraszewski, and S. O. Nelson, Nondestructive microwave characterization for determining the bulk density and moisture content of shelled corn, Meas. Sci. Technol., vol. 9, pp. 1548 - 1556, 1998. 